\title{
Implementation of a Diagnostics Consultation Service Improves Health Outcomes
}

Consultations with healthcare practitioners and consumers provide clinical laboratory scientists opportunities for evidence-based assessment of the impact of diagnostic information on health outcomes. Methods for characterization and impact of CLS consultations on health outcomes have not been reported. To address the role of CLS consultations in clinical decision support (CDS), consultation services have been established and evaluated in four healthcare delivery settings: consumer information $(\mathrm{Cl})$, diagnostic management intervention (DMI), patient care rounding intervention (PCRI), and utilization review (UR). Cl involve interaction with consumers with questions about interpretation of diagnostics parameters and results. DMI are primarily inpatient and requiring hand-offs among multiple providers. PCRI can be simple or complex involving literature searches using hand-held devices and interaction with team members. UR is driven by laboratory information system data generated by locally-generated and published rules defining daily reports of errors and inappropriate test orders. Consultation interactions in each setting are characterized by variables: provider type, medical subject/diagnosis, diagnostic question, testing cycle phase, treatment phase (screen/monitor/diagnose), complexity (number of hand-offs and/or logic steps, and patient and financial outcomes. The data collected for each variable were analyzed from these encounters over a one-year period. From these consultations, priority for direction of clinical laboratory resources (material/human) and value (quality/cost) of information have been established. Implementing the Consultation Model, real time evidence from consultation services is combined with evidence from the literature, used to monitor for and correct patient safety concerns, and inform efforts to provide accurate, timely information for CDS and shared decision-making between consumers and healthcare providers. 